\title{
Preparation and characterization of novel, mucoadhesive ofloxacin nanoparticles for ocular drug delivery
}

\author{
Shiva Taghe ${ }^{1,2}$, Shahla Mirzaeei ${ }^{3,4}$ \\ ${ }^{1}$ Student Research Committee School of Pharmacy, Kermanshah University of Medical Sciences, Kermanshah, Iran, \\ ${ }^{2}$ Department of pharmaceutical chemistry, Faculty of Pharmaceutical Chemistry, Pharmaceutical Sciences Branch, (IAUPS), \\ Tehran, Iran, ${ }^{3}$ Pharmacutical Sciences Research Center, School of Pharmacy, Kermanshah University of Medical Sciences, \\ Kermanshah, Iran, ${ }^{4}$ Nano Drug Delivery Research Center, School of Pharmacy, Kermanshah University of Medical Sciences, \\ Kermanshah, Iran
}

\begin{abstract}
The efficacy of conventional ocular formulations is limited by poor corneal retention and permeation, resulting in low ocular bioavailability. Mucoadhesive chitosan (CS)/ tripolyphosphatesodium (TPP) and chitosan (CS)/ tripolyphosphatesodium (TPP)-alginate (ALG) nanoparticles were investigated for the prolonged topical ophthalmic delivery of ofloxacin. A modified ionotropic gelation method was used to produce ofloxacin-loaded nanoreservoir systems. The ofloxacin-loaded CS/TPP and CS/TPP-ALG nanoparticles were characterized for particle size, morphology, zeta potential, encapsulation efficiency, subsequent release and corneal penetration study. The designed nanoparticles have a particle size from $113.8 \mathrm{~nm}$ to $509 \mathrm{~nm}$ and zeta potential from $16.2 \mathrm{mV}$ to $40.3 \mathrm{mV}$ and encapsulation efficiency values ranging from $19.7 \%$ to $33.1 \%$. Nanoparticles revealed a release during the first hours, followed by a more gradual drug release. The ofloxacin-loading CS/TPP or CS/TPP-ALG NPs developed are pronounced penetration enhancing effect as compared to OFX solution (5-6.5 times). Thus, these nanoparticles have a strong potential for ocular drug delivery.
\end{abstract}

Keywords: Ocular drug delivery. Nanoparticles. Corneal penetration. Ofloxacin. Chitosan.

\section{INTRODUCTION}

Infections of the eye are caused by a number of different organisms and require effective treatment to avoid further complications and vision loss. Systemic treatment is not ideal due to systemic side effects and the low ocular bioavailability of the drug (Duxfield et al., 2016). The eye is a unique organ for drug delivery. According to the eye's physiology and anatomy, a small percentage of prescription drug was absorbed, because the protection mechanisms, such as tearing, blinking reflex and tears streaming from the eyes, are expelled (Thakur, Kashiv, 2011). About $5 \%$ of the eye drugs in the cornea reaches the intraocular tissues, while a major fraction of the instilled dose is often absorbed systemically via the conjunctiva and nasolacrimal duct (Lang, 1995). The more topical aqueous solution and suspension forms after use impair vision.

*Correspondence: Shahla Mirzaeei. Pharmacutical Sciences Research Center, Health Institute, Kermanshah University of Medical Sciences, Kermanshah, Iran. E-mail:shahlamirzaiie@gmail.com (iD
But a rapid and short-input entry of some drugs into the systemic circulation is unavoidable, and this formulation is quickly diluted by the tear layer (Nagarwal et al., 2009). Nowadays, more sensitive diagnostic techniques and novel therapeutic agents are used to provide ocular delivery systems with high therapeutic efficacy. Nanoparticles show a better application as compared to conventional delivery systems (Das, Suresh, 2010). Proposed polymer nanoparticles are reported to be devoid of any irritant effect on the cornea, iris, and conjunctiva, and thus appear to be suitable inert carriers for ophthalmic drug delivery. Specific nanocarriers can interact with the ocular mucosa, thereby increasing the retention time of drug in the eye, and its permeability across the cornea and conjunctiva (Reimondez-Troitiño et al., 2015). Taking into account this information and also the fact that the cornea and conjunctiva have a negative charge, it was thought that the use of mucoadhesive polymers that might interact intimately with these extra ocular structures would increase the concentration and residence time of the associated drug (Sahoo, Dilnawaz, Krishnakumar, 2008). 
Among the mucoadhesive polymers investigated until now, the cationic polymer chitosan (CS) has attracted a great deal of attention because of its unique properties, such as acceptable biocompatibility and biodegradability (Hirano et al., 1988) as well as the ability to increase membrane permeability (Aspden et al., 1997; Takeuchi et al., 1996). The substrate specificities of lysozyme in the degradation of soluble chitosan are rather independent for $\mathrm{pH}$ between 4.5 and 7, and it may be inferred that these substrate specificities also operate in vivo at physiological conditions (Vårum et al., 1997). The interaction and prolonged residence time of CS nanoparticles at the ocular mucosa of rabbits have been reported, and it was shown that following topical instillation of fluorescence-labelled nanoparticles, these colloidal drug carriers remained attached to the cornea and the conjunctiva for at least 24 h (De Campos, Sánchez, Alonso, 2001). The presence of CS in an ophthalmic solution resulted in a significant increase of the precorneal residence time of antibiotic drugs when compared with commercial drug solutions (Felt et al., 1999). Ofloxacin and chitosan alone have an antimicrobial effect against both gram-positive and gram-negative bacteria, but complexation (CS-OFX) is better enhanced by the antimicrobial activity (Singh, Dutta, 2010). Sodium alginate was chosen as a vehicle for ophthalmic formulations since it exhibits several favourable biological properties such as biodegradability and non-toxicity. A prolonged precorneal residence of formulations containing alginic acid was looked for, not only because of its ability to gel in the eye, but also due to its mucoadhesive properties (Sechoy et al., 2000). Ofloxacin was successfully formulated as an ion-activated in situ gel forming ophthalmic solution using sodium alginate in combination with Hydroxy Propyl Cellulose (HPC) as a viscosity enhancer, which sustained the drug release over a period of $8 \mathrm{~h}$ (Abraham et al., 2009). Chitosanalginate (CS-ALG) polyionic complexes protecting the encapsulate limit the release of encapsulated materials more effectively than either alginate or chitosan alone (Yan, Khor, Lim, 2001). Ofloxacin is as an agent against external ocular infections, and is exceptionally potent against a wide range of gram-positive and gram-negative bacteria and obligate anaerobes. It is effective in vitro against many organisms that cause ocular infections, for example haemophilus influenzae, neisseria gonorrhoeae, staphylococcus species, streptococcus species and pseudomonas aeruginosa, which are associated with ocular infections that are especially serious and difficult to treat (Bron et al., 1991). The excellent activity of ofloxacin, its broad spectrum of activity, its high tear concentrations, and its reported clinical efficacy all support its potential use as a treatment for ocular bacterial infections (Osato et al., 1989). The current study is aimed at developing and optimizing a mucoadhesive nanoparticulate formulation of ofloxacin for ocular delivery.

\section{MATERIAL AND METHODS}

\section{Material}

Low molecular weight chitosan (CS) with a degree of deacetylation of $85 \%$ and tripolyphosphate sodium (TPP) obtained from Sigma-Aldrich (USA). Acetic acid at $100 \%$ was purchased from Merk (Darmstadt, Germany). The medium viscosity sodium alginate (ALG) was purchased from Sigma-Aldrich (USA). Ofloxacin (OFX) was provided Exir (Lorestan, Iran). Sodium hydroxide $(\mathrm{NaOH})$ and hydrochloric acid $(\mathrm{HCl})$ were from Merk (Darmstadt, Germany). Methanol and triethylamine were of analytical grade and purchased from Merck (Darmstadt, Germany). All other materials and reagents were of the highest grade commercially available.

\section{Methods}

\section{Preparation of Nanoparticles without ALG (Chitosan/ tripolyphosphatesodium nanoparticles)}

Chitosan/tripolyphosphatesodium nanoparticles (CS/TPP NPs) were prepared by an ionic gelation method as reported by Calvo et al. (1997) with some modifications. $\mathrm{CS}$ was dissolved in acetic acid $(1 \% \mathrm{v} / \mathrm{v})$ to obtain the cationic phase $(100 \mathrm{~mL})$ in different concentrations. CSNPs were obtained upon the addition of $2 \mathrm{~mL}$ of TPP solution in two respective final concentrations ( 0.5 and $0.75 \mathrm{mg} / \mathrm{mL}$ ) by drop-wise to $5 \mathrm{~mL}$ of chitosan solution under magnetic stirring $(1000 \mathrm{rpm})$ at room temperatures for $20 \mathrm{~min}$. The experimental flow sheet is shown in Figure 1. For the preparation of OFX-loaded nanoparticles, 100 $\mathrm{mg}$ of ofloxacin was dissolved in the cationic phase (100 $\mathrm{mL}$ ) and the final nanoparticle suspension was prepared as described earlier. In order to achieve the final OFX:CS mass ratios of 1:0.5, 1:1.5, 1:3.5, and 1:5, four formulations $(\mathrm{Fa}, \mathrm{Fb}, \mathrm{Fc}$ and $\mathrm{Fd}$ ) with different concentrations of $\mathrm{CS}$ and TPP were prepared as shown in Table I.

\section{Preparation of nanoparticles with ALG (chitosan/ tripolyphosphatesodium-alginate) nanoparticles)}

The sodium alginate solutions in concentration $(0.05,0.1,0.25$ and $0.5 \mathrm{mg} / \mathrm{mL})$ were prepared by dissolving the polymer in $100 \mathrm{~mL}$ of TPP solution in concentration $(0.75 \mathrm{mg} / \mathrm{mL})$. Ofloxacin-loaded NPs were formed spontaneously upon drop-wise addition of $2 \mathrm{~mL}$ of 


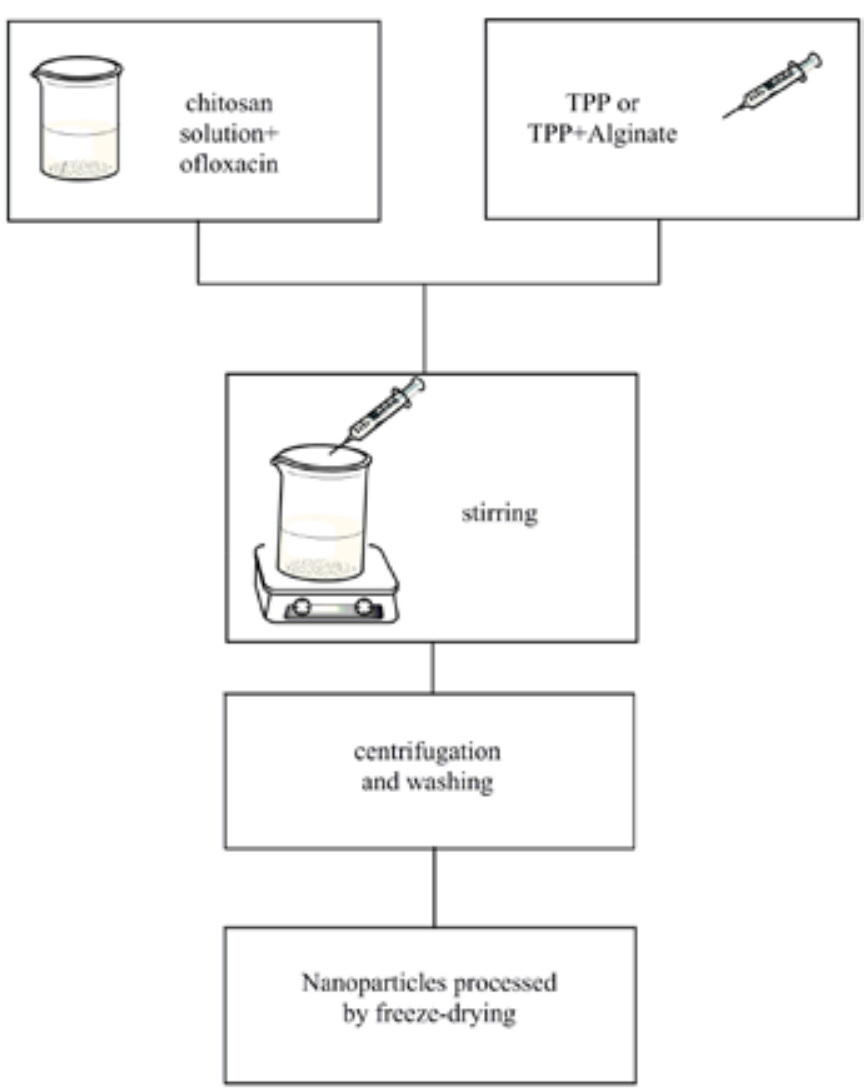

FIGURE 1 - The experimental procedure.

TPP-ALG solution to $5 \mathrm{~mL}$ of chitosan solution $(1.5,3.5$ $\mathrm{mg} / \mathrm{mL})$ containing ofloxacin $(1 \mathrm{mg} / \mathrm{mL})$ with magnetic stirring $(1000 \mathrm{rpm})$ for $20 \mathrm{~min}$ at room temperature. In order to achieve final OFX:Polymers mass ratios of 1:1.54, 1:1.54, 1:3.6, 1:3.7, four formulations ( $\mathrm{Fe}, \mathrm{Ff}, \mathrm{Fg}$ and $\mathrm{Fh}$ ) with different $\mathrm{Cs} / \mathrm{TPP} / \mathrm{ALG}$ mas ratio were prepared as shown in Table I.

Collecting of solid state chitosan particulate by freeze drying

The nanoparticle suspensions centrifuged at 30,000 rpm for 30 min (Beckman Coulter centrifuges, optimaL90k, USA), were used respectively. The nanoparticles were removed, and these suspensions were frozen and then freeze-dried (Christ Freeze Dryer ALPHA 2-4, PLUS, GERMANY). The dried residues were stored at $4^{\circ} \mathrm{C}$ until analysis.

\section{Determination of encapsulation efficiency and the particles' drug content}

The encapsulation efficiency of OFX-loaded NPs was calculated as the difference between the total amount of ofloxacin used to prepare the nanoparticles and the amount of ofloxacin present in the supernatant after centrifugation of the nanoparticle suspensions (Wu et al., 2005). The amount of drug was determined by an HPLC method as described in the section of HPLC method. The drug content (DC) and the encapsulation efficiency (EE) were calculated by using the following equations (Equations (1) and (2) respectively):

$$
\begin{aligned}
& \text { \% The drug content }=\frac{\text { Total amount of ofloxacin }- \text { Free drug }}{\text { Weight of nanoparticle }} \\
& \% \text { Encapsulation efficiency }=\frac{\text { Total amount of ofloxacin }- \text { Free drug }}{\text { Total amount of ofloxacin }}
\end{aligned}
$$

\section{Particle size, PDI and zeta potential measurements}

The particle size and the zeta potential of the freshly prepared NPs were determined by photon correlation spectroscopy and laser-doppler anemometry by using a Malvern Zetasizer, and the nanoparticle suspensions were analysed at $25^{\circ} \mathrm{C}$. Each sample was measured three times, after which the average value was considered for the data analysis of zeta potential.

\section{HPLC method}

Chromatographic separations were performed by using a Shimadzu (model LC-10ADvp) liquid chromatography connected to a UV-VIS detector (model SPD-M10Avp) and to a ChromatoPlus computerized integration system (Shimadzu Corporation, Kyoto, Japan). Manual injections of the samples were performed by using a Rheodyne 7725 injector with a $20 \mu \mathrm{L}$ sample loop. The analytical column $(250 \times 4.6 \mathrm{~mm}$ I.D. $)$ was packed with C18 (5 $\mu \mathrm{m}$ particle size) by MZ-Analysentechnik (Mainz, Germany). Methanol and triethylamine $0.1 \%$ (TEA $0.1 \%$ ) buffer at $\mathrm{pH} 3.0$ with phosphoric acid $(10: 90 \mathrm{v} / \mathrm{v})$ were used as the mobile phases (flow rate of $1.2 \mathrm{~mL} / \mathrm{min}$ ). Ultraviolet detection was set at $293 \mathrm{~nm}$ and the elution time was $2.4 \mathrm{~min}$.

\section{Stability of nanoparticles}

To understand the stability of nanoparticles in suspension, two formulations of nanoparticles Fc and Fg were chosen after their preparation, nanoparticle suspension was lyophilized using 5\% mannitol as a cryoprotectant. Dry nanoparticles were suspended in phosphate buffer saline; particle size, and zeta potential measurements were performed. Freeze-dried samples, stored at room temperature, were rehydrated with the 
original volume of ultrapure water to restore the drug and polymer concentrations at every 30 days interval and the particle size and zeta potential measurements were taken. Three measurements of particle size and zeta potential were performed and used in statistical analysis of the data (Agnihotri, Aminabhavi, 2007; Motwani et al., 2008)

\section{Fourier transform infrared (FTIR) spectral studies}

FTIR spectra were obtained by using an FTIRspectrometer (Shimadzu IR PRESTIGE-21., Japan). The samples were dried in a vacuum desiccator, mixed with micronized $\mathrm{KBr}$ powder and compressed into discussing a manual tablet press. The FTIR spectra of pristine ofloxacin, ALG, Chitosan, Chitosan/TPP NPS and Chitosan/TPP-ALG nanoparticles were obtained.

\section{Thermo-gravimetric studies}

TGA analysis was obtained using (STA 503, BAHR, Germany). $5.0 \mathrm{mg}$ of the freeze-dried powder samples crimped in a standard platinum pan and heated from 20 to $500{ }^{\circ} \mathrm{C}$ with a nitrogen flow rate of $6 \mathrm{~L} \cdot \mathrm{h}^{-1}$ and a heating rate of $10^{\circ} \mathrm{C} / \mathrm{min}$.

\section{Scanning Electron Microscopic (SEM) Studies}

Scanning electron microscopy (SEM) was used to observe the morphology of the nanoparticles. The NPs were dried at room temperature and placed on metal stubs with adhesive tape, sputter coated with gold and then observed under a scanning electron microscope (KYKY, EM-3200, China) operating in high vacuum moderated at an accelerating voltage of $20 \mathrm{kV}$.

\section{Drug release study}

To detect the amount of drug released from the nanoparticles, an appropriate amount of sample (estimated to contain approx. $5 \mathrm{mg}$ of NPs or ofloxacin control solution) was introduced into an acceptor compartment containing $1 \mathrm{~mL}$ of phosphate buffer at $\mathrm{pH} 7.4$ with sink condition, respectively, separated by a dialysis membrane (Mw cut-off $=12,000-14,000$ Daltons; Delchimica Scientific Glassware, Milan, Italy), as illustrated in Figure 2(A), from the receptor compartment containing $9 \mathrm{~mL}$ of the same aqueous buffer. The system was stirred continuously at $100 \mathrm{rpm}$ maintained at $37^{\circ} \mathrm{C}$. The receptor compartment was closed to prevent evaporation losses from the dissolution medium as shown in Figure2(C). A certain amount of sample aliquot was withdrawn at regular time intervals and the same volume was replaced with a fresh buffer and the drug concentration was quantified in the acceptor phase by high performance liquid chromatography (HPLC) following the method previously described in the section of HPLC method.
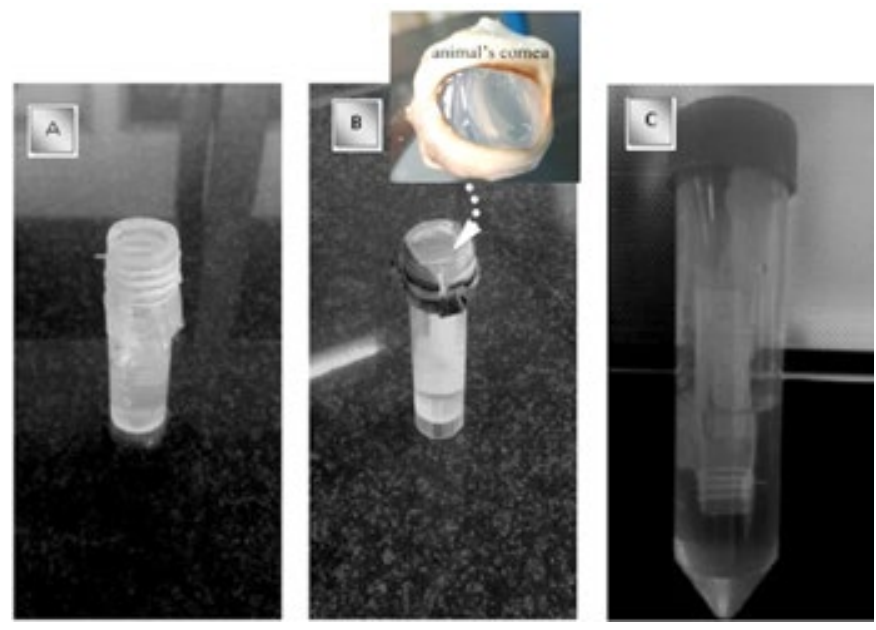

FIGURE 2 - The acceptor compartment for drug release (A), the acceptore compartment for penetration studies (B), receptor compartment (C).

\section{Ofloxacin penetration study through isolated sheep corneas}

Corneal and conjunctival epithelia are the key tissues in topical ocular absorption of drugs. These tissues contain tight junctions that limit the paracellular drug (Maurice, Mishima, 1984). Cornea is a tight barrier for drug absorption. Permeability of the corneal epithelium is $10^{-7}-10^{-5} \mathrm{~cm} / \mathrm{s}$ and the bioavailability of ocular administration is very low (Urtti et al., 1990). In addition, the corneal epithelium have mucus layer on their surface which protects all wet epithelial surfaces.

For the penetration study, using excised sheep corneas. Within $1 \mathrm{~h}$ after the euthanasia, a small transverse incision was made about $5 \mathrm{~mm}$ from the limbus, and subsequently the cornea with the scleral ring was carefully cut out. Forceps were used to delicately remove the lens first and then the iris, with the cornea being left as a transparent film. The cornea was then mounted as maintained on the cornea curvature. A volume of $1 \mathrm{~mL}$ PBS (pH 7.4) with $5 \mathrm{mg}$ NPs or OFX control solution were added first in the acceptor compartment, as shown in Figure 2(B), at $37^{\circ} \mathrm{C}$ to and then the cornea was fixed in and the epithelial side was immersed in PBS at the receptor compartment. A certain amount of samples were taken from the receptor compartment at certain intervals 
and then the sample volume was immediately replaced with an equal amount of PBS buffer, in order to maintain receptor compartment conditions. The amount of OFX in the acceptor was determined by using the HPLC method as described in the section of HPLC method. The code of ethics approved for this project is IR.KUMS. REC.1395.216.

\section{Calculation of permeability coefficients}

The apparent corneal permeability coefficient (Papp, $\mathrm{cm} . \mathrm{s}^{-1}$ ) was determined according to

$$
\operatorname{Papp}=\Delta \mathrm{Q} / \Delta \mathrm{tAC}_{0}
$$

where $\mathrm{Q}$ is the total amount permeated at time t, $\Delta \mathrm{Q} / \Delta \mathrm{t}$ (the slope of the linear portion of the graph) is the steadystate flux of ofloxacin to the receiver side $(\mu \mathrm{g} / \mathrm{min}), \mathrm{A}$ is the corneal surface area (in this study $1 \mathrm{~cm}^{2}$ ) and $\mathrm{C}_{0}$ is the initial donor side drug amount $(\mu \mathrm{g} / \mathrm{mL})$ (Calvo et al., 1996).

When the steady-state portion of the drug permeated VS time graph is extrapolated, the point of intersection at the time axis gives the lag time of permeation $\left(t_{L}\right)$. This is the time required to establish a steady concentration gradient within the membrane separating the donor from the receptor compartment.

\section{RESULTS AND DISCUSSION}

\section{Preparation of CS/TPP and CS/TPP-ALG nanoparticles}

In this study, we described the preparation of a chitosan nanoparticulate system that is able to incorporate ofloxacin, with appropriate mucoadhesiveness and antimicrobial characteristics for the treatment of ocular infections. Chitosan nanoparticles were produced by inotropic gelation, a method based on the formation of complexes between chitosan and TPP or chitosan and TPPALG, under mild conditions. In this method, nanoparticles are formed spontaneously upon mixing of CS and TPP or TPP-ALG solutions, through the formation of inter- and intra-molecular linkages between the phosphate groups of TPP and the amino groups of Chitosan (Fan et al., 2012) and interactions between the carboxyl groups of alginate and the amine groups of chitosan (Yan, Khor, Lim, 2001). As reported in the literature, the formation process of the nanoparticles is influenced by a number of parameters such as CS,TPP and ALG concentrations, and the time and rate of mixing. Therefore, preliminary experiments were performed to optimize CS/TPP and CS/TPP-ALG ratios for nanoparticle preparation through visual observation of the dispersions and size testing. Finally, the various formulations with different mass ratios of CS/TPP or CS/ TPP-ALG were prepared as shown in Table I.

\section{Determination of particle size, PDI and zeta potential}

The size, polydispersity index (PDI) and zeta potential of NPs are reported in Table II. The mean particle size was in the range of 113-509 $\mathrm{nm}$. The particle size, as previously reported by Calvo et al., (Calvo, Remunan-Lopez et al., 1997), depends on the CS concentration. The minimum size corresponds to the lowest CS concentration ( $\mathrm{Fa})$. On the other hand, the size of the particles was affected by the Alginate concentration (Motwani, Chopra et al., 2008), with the size being smaller for lower ALG concentration. These results confirm that smaller NPs are the result, when the availability of the functional groups on two

TABLE I - Preparative characteristics of the different formulations

\begin{tabular}{ccccc}
\hline Formulation & CS (mg/mL) & TPP $(\mathbf{m g} / \mathbf{m L})$ & ALG $(\mathbf{m g} / \mathbf{m L})$ & $\begin{array}{c}\text { CS:TPP } \\
\text { mass ratio }\end{array}$ \\
\hline Fa & 0.5 & 0.5 & - & $1: 0.4$ \\
Fb & 1.5 & 0.5 & - & $1: 0.13$ \\
Fc & 3.5 & 0.75 & - & $1: 0.086$ \\
Fd & 5 & 0.75 & - & $1: 0.06$ \\
Fe & 1.5 & 0.75 & 0.05 & $1: 0.2$ \\
Ff & 1.5 & 0.75 & 0.1 & $1: 0.2$ \\
Fg & 3.5 & 0.75 & 0.25 & $1: 0.086$ \\
Fh & 3.5 & 0.75 & 0.5 & $1: 0.086$ \\
\hline
\end{tabular}


polymers for interaction is in stoichiometric proportion. The zeta potential of the nanoparticles ranged between +16.2 and $+40.3 \mathrm{mV}$, which shows a dependency on the concentration of CS. A gradual increase in the zeta potential was noted with the increase in CS concentration and the reduction in ALG concentration. It can be ascribed to the higher availability of protonated amine groups with increasing CS concentration. There is no relationship between CS concentration and PDI of the nanoparticles that were formed by CS and TPP. However, the PDI of NPs were increased by increasing alginate concentration in CS/ TPP-ALG nanoparticles (Table II).

\section{Determination of encapsulation efficiency and the particles' drug content}

The observations for encapsulation efficiency (\%) and the particles'drug content (\%) are presented in Table II. The increasing concentration of CS from $0.5 \mathrm{mg} / \mathrm{mL}$ to $1.5 \mathrm{mg} / \mathrm{mL}$ at a constant TPP and drug concentration caused a great increase in the encapsulation of ofloxacin into CS/TPP nanoparticles from $19.78 \%$ to $28.62 \%$, and the increasing concentration of CS from $3.5 \mathrm{mg} / \mathrm{mL}$ to $5 \mathrm{mg} / \mathrm{mL}$ also caused a great increase in the encapsulation efficiency from $24.06 \%$ to $33.1 \%$. This indicates that less amount of nanoparticles were formed due to the lower chitosan concentration. It was also observed by Motwani et al. (2008) that the encapsulation of gatifloxacin into CS/ALG nanoparticles was highest when CS and ALG were used at intermediate concentrations, whereas the encapsulation was found to be fewer when either CS or ALG was used at higher concentration levels (Motwani et al., 2008).

Also, the CS/TPP-ALG nanoparticles showed a slight increase in encapsulation efficiency and drug content with respect to that of CS/TPP nanoparticles. This could be due to an increase in crosslink density, which might prevent the leaching of drug particles during the formation of nanoparticles.

\section{Morphology}

Morphological characterizations via scanning electron microscopy (Figure 3) showed nanoparticles exhibiting rounded and some oblate shapes. The shapes of the particles were approximate to spheres, and were smooth with almost homogeneous structures. Moreover, the nanoparticles tended to agglomerate probably due to the experimental conditions used for the preparation of NPs. CS/TPP NPs were nearly spherical in shape and smaller in size (Figure 3A), while these nanoparticles' structure took a regular shape and larger size after addition of ALG (Figure 3B).

\section{Identification of nanoparticles constituents}

CS, ALG, ofloxacin, CS/TPP nanoparticles, and CS/TPP-ALG nanoparticles were analysed using an FTIR spectrophotometer for characteristic absorption bands, indicating their interactions (Figure 4). The FTIR spectrum of CS shows characteristic peaks at $\sim 1072 \mathrm{~cm}^{-1}$ (due to the presence of -CO groups) and at $\sim 3400 \mathrm{~cm}^{-1}$ (representing the presence of $\mathrm{OH}$ groups) and at $\sim 1600 \mathrm{~cm}^{-1}$ in the CS spectra due to the $-\mathrm{NH} 2$ groups of CS. In the IR spectrum of the CS/TPP and CS/TPP-ALG nanoparticles, this peak shifted from $\sim 1600 \mathrm{~cm}^{-1}$ to $1624 \mathrm{~cm}^{-1}$, thus confirming that amino groups were involved in the cross-linking by phosphate. The IR spectrum of ofloxacin showed its characteristic peaks at $\sim 1000 \mathrm{~cm}-1$ for $\mathrm{C}-\mathrm{F}, 1712 \mathrm{~cm}^{-1}$ for $\mathrm{C}=\mathrm{O}$, and $3425 \mathrm{~cm}^{-1}$ for $\mathrm{OH}$ (Sahoo et al., 2012). The shift of the $\mathrm{C}=\mathrm{O}$ peak at $1712 \mathrm{~cm}^{-1}$ confirmed the presence of ofloxacin inside both the CS/TPP and CS/TPP-ALG

TABLE II - Properties of the different formulations (mean $\pm \mathrm{SD}, \mathrm{n}=3$ )

\begin{tabular}{cccccc}
\hline Formulation & Size (nm) & PDI & $\begin{array}{c}\text { Zeta Potential } \\
(\mathbf{m v})\end{array}$ & EE\% & DC\% \\
\hline Fa & $113.8 \pm 2.3$ & $0.252 \pm 0.08$ & $16.2 \pm 3.6$ & $19.78 \pm 0.13$ & $19.78 \pm 0.19$ \\
Fb & $165.5 \pm 3.9$ & $0.257 \pm 0.23$ & $23.8 \pm 2.7$ & $28.62 \pm 0.21$ & $23.85 \pm 0.23$ \\
Fc & $287.4 \pm 4.6$ & $0.242 \pm 0.19$ & $30.6 \pm 2.1$ & $24.06 \pm 0.16$ & $15.32 \pm 0.17$ \\
Fd & $396.1 \pm 2.9$ & $0.254 \pm 0.11$ & $40.3 \pm 1.8$ & $33.1 \pm 0.22$ & $16.32 \pm 0.09$ \\
Fe & $227.6 \pm 1.6$ & $0.313 \pm 0.17$ & $28.1 \pm 1.2$ & $29.88 \pm 0.53$ & $28.46 \pm 0.14$ \\
Ff & $243.1 \pm 4.3$ & $0.373 \pm 0.32$ & $32.4 \pm 3.2$ & $29.77 \pm 0.34$ & $23.63 \pm 0.1$ \\
Fg & $439.8 \pm 1.9$ & $0.39 \pm 0.14$ & $37.4 \pm 1.7$ & $24.82 \pm 0.43$ & $18.66 \pm 0.08$ \\
Fh & $509 \pm 5.2$ & $0.423 \pm 0.28$ & $35.3 \pm 2.6$ & $22.95 \pm 0.24$ & $17.08 \pm 0.09$ \\
\hline
\end{tabular}




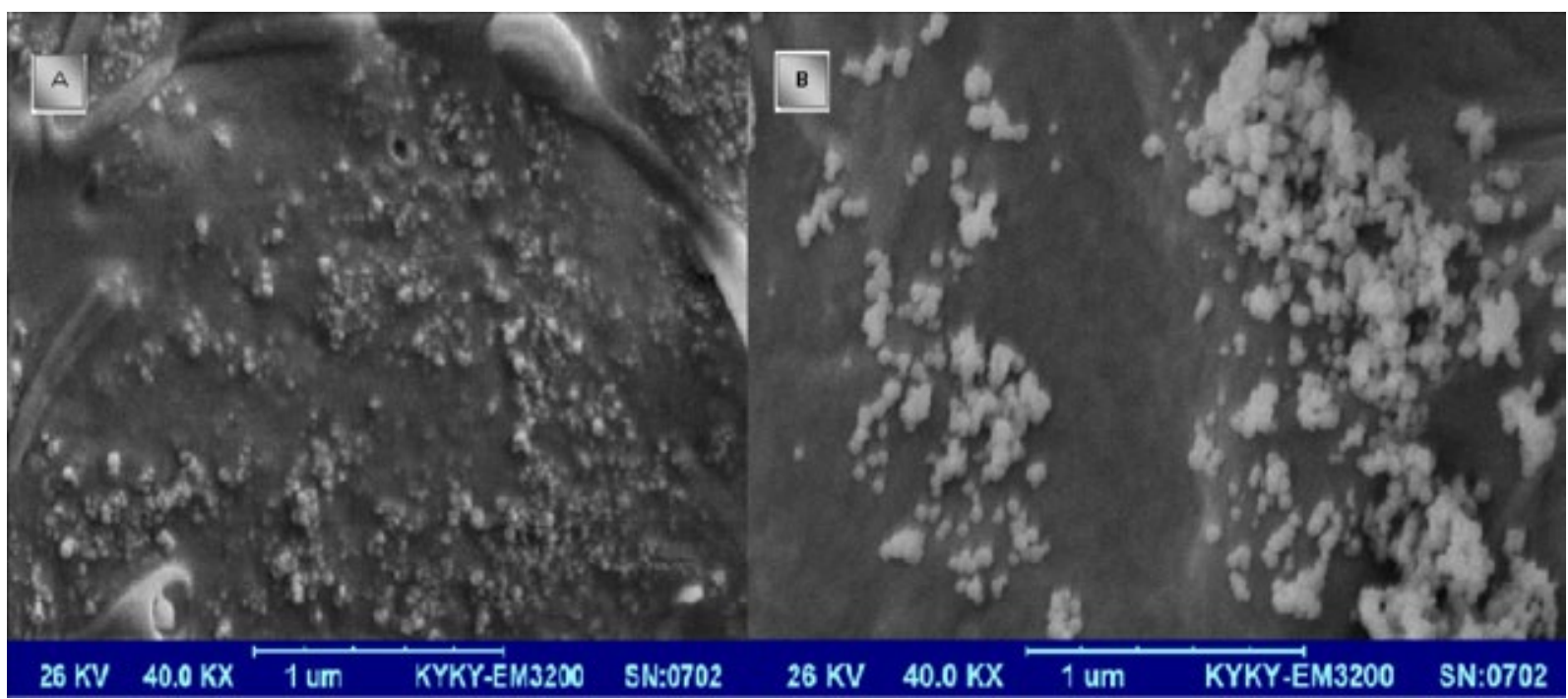

FIGURE 3 - Scanning electron micrographs of Chitosan /TPP NPs (A), Chitosan /TPP-Alginate NPs (B), bar represent $1 \mu \mathrm{m}$.

nanoparticle structure. The IR spectrum of ALG showed its characteristic peaks at $3425 \mathrm{~cm}^{-1}$ for $\mathrm{OH}, 948 \mathrm{~cm}^{-1}$ for O-H bend (out-of-plane) and $1419 \mathrm{~cm}^{-1}$ and 1616 $\mathrm{cm}^{-1}$ for symmetric and asymmetric stretching of $\mathrm{COO}^{-}$ (Papageorgiou et al., 2010). The disappearance of peaks at $1419 \mathrm{~cm}^{-1}$ and $1616 \mathrm{~cm}^{-1}$, and also the observation of the peak at $1490 \mathrm{~cm}^{-1}$ in the FT-IR spectrum of CS/TPPALG nanoparticles was attributed to the ionic interactions between the carboxyl groups of alginate and the amine groups of chitosan.

\section{Thermo-gravimetric studies}

The TGA curves of pristine ofloxacin, CS, ALG, CS/TPP NPS and CS/TPP-ALG NPS are presented in Figure 5. There are two distinct changes in the TG profiles of ofloxacin. The first weight loss from $25^{\circ} \mathrm{C}$ to $150^{\circ} \mathrm{C}$ may be due to free water evaporation. The second weight loss from $289^{\circ} \mathrm{C}$ to $395^{\circ} \mathrm{C}$ is attributed to OFX decomposition. In the curves of CS, the first weight loss from $25^{\circ} \mathrm{C}$ to $100^{\circ} \mathrm{C}$ may be attributed to free water evaporation. The

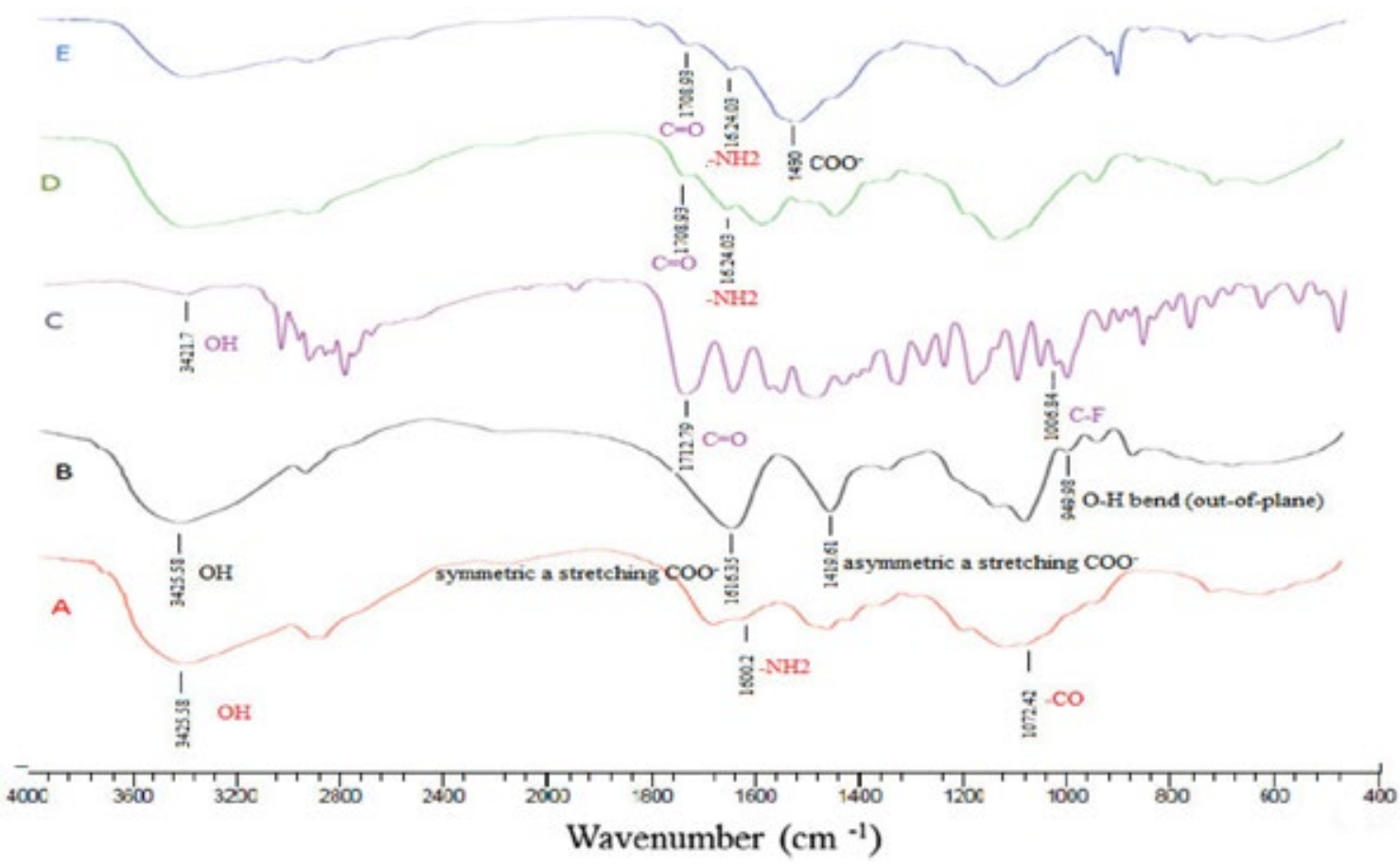

FIGURE 4 - FTIR spectrum: Chitosan (A); Alginate (B); Ofloxacin (C); Chitosan/TPP nanoparticles (D); Chitosan/TPP-Alginate nanoparticles (E). 
second weight loss from $273^{\circ} \mathrm{C}$ to $303^{\circ} \mathrm{C}$ may be due to $\mathrm{CS}$ decomposition. In the curves of ALG, the weight loss from $235^{\circ} \mathrm{C}$ to $275^{\circ} \mathrm{C}$ corresponds to the decomposition of ALG. The other weight losses are the same as those of ofloxacin and CS. The TG patterns of CS/TPP NPs are shown in Figure 6(D). The weight loss from $223^{\circ} \mathrm{C}$ to $296^{\circ} \mathrm{C}$ may be attributed to the destruction of ionic bond between CS and TPP and disintegrated nanoparticles. We can see that disintegrated nanoparticles come out around $249^{\circ} \mathrm{C}$ to $348^{\circ} \mathrm{C}$ in Figure 6(E), which may be explained by the fact that the ALG is partly encapsulated into the nanoparticles. The last weight losses from $309^{\circ} \mathrm{C}$ to $451^{\circ} \mathrm{C}$ in Figure 6(D) and from $353^{\circ} \mathrm{C}$ to $464^{\circ} \mathrm{C}$ in Figure 6(E) may be due to the destruction of drug decomposition.

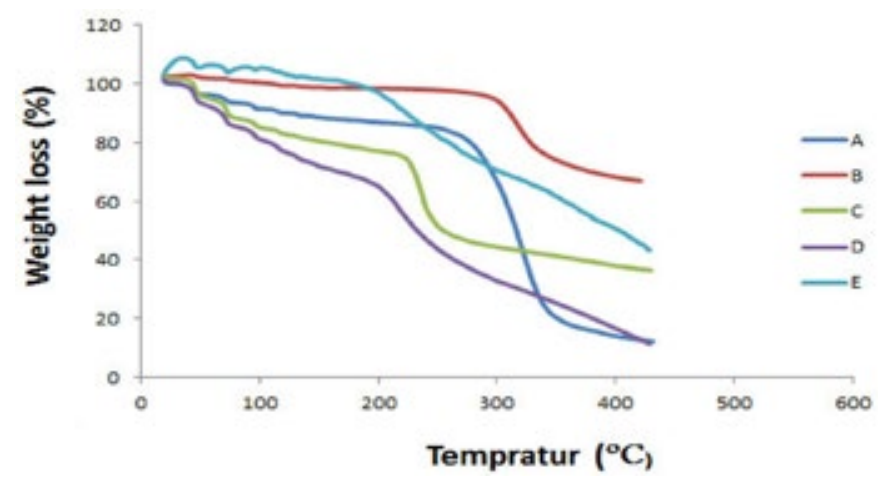

FIGURE 5 - TGA curves of Ofloxacin (A); Chitosan (B); Alginate (C); Chitosan/TPP nanoparticles (D); Chitosan/TPPAlginate nanoparticles (E).

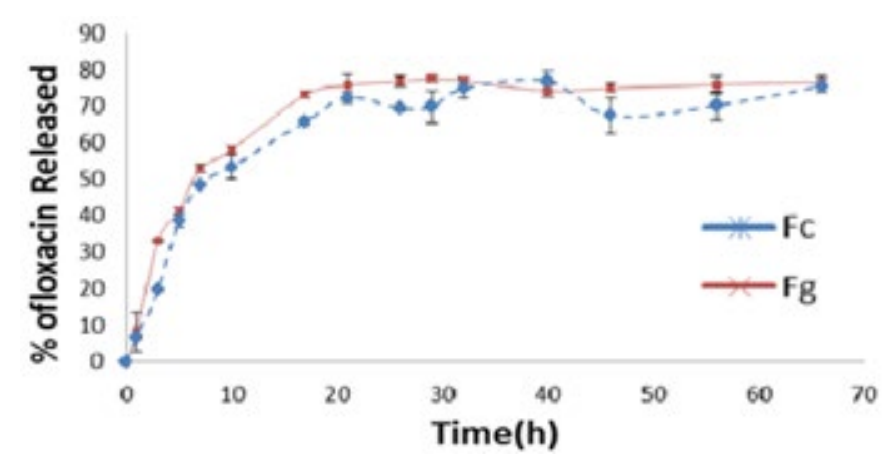

FIGURE 6 - In vitro release profile of ofloxacin from Chitosan/ TPP nanoparticles (Fc); Chitosan/TPP-Alginate nanoparticles (Fg). Release assay was performed in PBS solution, pH: 7.4, at $37^{\circ} \mathrm{C}$ with agitation $(\mathrm{n}=3)$.

\section{In vitro ofloxacin release study}

The results of OFX release from CS nanoparticles are summarized in Figure 6. For Fc formulation, more than $50 \%$ of drug content ( $\% 53 / 5)$ was released in $10 \mathrm{~h}$ with the drug release peak in $20 \mathrm{~h}$, and a small amount of OFX was released after 20 to $66 \mathrm{~h}$. In 7 hours, the drug content of Fg was released (52.9\%), and reached $73.1 \%$ within $17 \mathrm{~h}$ of release. Both formulations had amounts of residual drug up to $66 \mathrm{~h}$. Comparative release indicated that chitosan nanoparticles were capable of releasing the drug for a long time, which this could be due to the structural strength of chitosan nanoparticles in the coating. The result demonstrated sustained/prolonged drug release from ofloxacin-loading CS/TPP or CS/TPP-ALG NPs.

\section{Ofloxacin penetration study through isolated sheep corneas}

The efficacy of nanoparticles to increase corneal penetration was assessed by measuring the transcorneal flux of the drug through isolated sheep cornea. Figure 7 shows the amount of OFX permeated from the NPs formulation and from the same concentration of OFX in solution. The results indicate that the OFX permeability coefficients in solution were 3.77 and $3.43\left(* 10^{-4} \mathrm{~cm} . \mathrm{s}^{-}\right)$ for OFX in solution and OFX in chitosan solution, which have no significant difference. This is because of the tight junctions among corneal epithelial cells. Also lag time for OFX in chitosan solution and OFX solution were 17 and $32 \mathrm{~h}$ respectively.

The percentage of drug penetration in Fc after 48 hours and in Fg after 40 hours has reached steady state. Nanoparticles formulation produced Papp, 18.9 and $25.45\left(* 10^{-4} \mathrm{~cm} . \mathrm{s}^{-1}\right)$ for $\mathrm{Fc}$ and $\mathrm{Fg}$, which were 5 and 6.7 times more than OFX in solution. This can be explained by the fact that $\mathrm{Fg}$ and $\mathrm{Fc}$ penetrated to the corneal surface by both electrostatic force and hydrogen bonds. On the other hand, the polycationic Fg and Fc could improve the permeability of cornea by opening the tight junctions among corneal epithelial cells, which was similar to the case of chitosan (Di Colo et al., 2004). In vivo experiments by M. De Campos et al. following topical instillation of CyA-loaded CS nanoparticles to rabbits, observed therapeutic concentrations in external ocular tissues (i.e., cornea and conjunctiva) during at least $48 \mathrm{~h}$ (De Campos, Sánchez, Alonso, 2001). Ocular penetration enhancers such as benzalkonium chloride, non-ionic surfactants, bile salts and EDTA have been widely investigated (Sultana et al., 2006), due to their nonspecific actions that include permanent damage to the ocular membranes and tight junction, and so they have a low safety profile.

\section{Stability}

For long-term storage of nanoparticles, aqueous solutions of the nanoparticles are basically required to be lyophilized powder as products and it must be reconstituted 


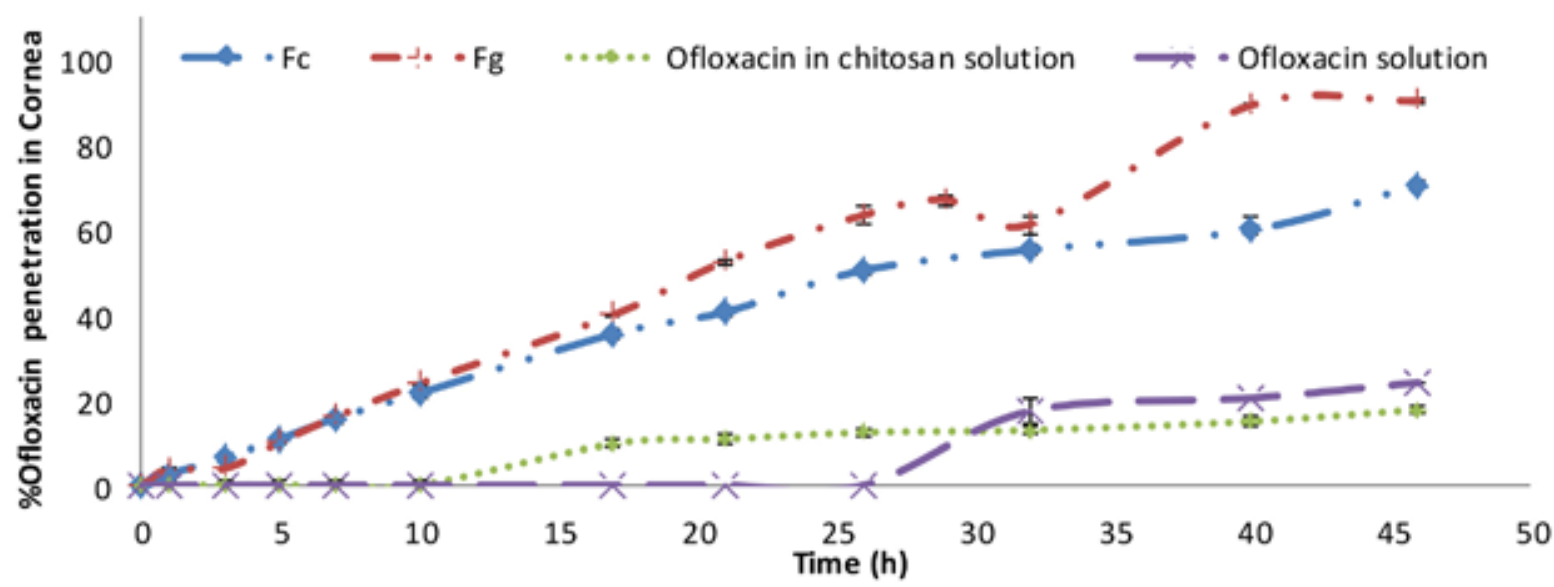

FIGURE 7 - In situe penetration profile of ofloxacin from Chitosan/TPP nanoparticles (Fc); Chitosan/TPP-Alginate nanoparticles $(\mathrm{Fg})$; ofloxacin in chitosan solution; ofloxacin solution. Penetration assay was performed in $\mathrm{PBS}$ solution, $\mathrm{pH}: 7.4$, at $37^{\circ} \mathrm{C}$ with agitation $(\mathrm{n}=3)$.

TABLE III - Permeability coefficients and lag time

\begin{tabular}{lcc}
\hline Formulation & Papp $\left(\times \mathbf{1 0}^{-4}\right)(\mathbf{c m} / \mathbf{s})$ & tL $(\mathbf{h})$ \\
\hline Ofloxacin solution & $3.77 \pm 0.235$ & 32 \\
Ofloxacin solution in chitosan & $3.43 \pm 0.311$ & 17 \\
Fc & $25.45 \pm 1.56$ & $<1$ \\
Fg & $18.9 \pm 0.560$ & $<1$ \\
\hline
\end{tabular}

into physiological solution similar to its original aqueous solution immediately before use (Motwani et al., 2008).
Figure 8 (CS/TPP) and Figure 9 (CS/TPP-ALG) show the change in particle size and the zeta potential of reconstructed nanoparticles suspension, after storage for a period of six months at room temperature. It showed that the size increased and the amount of zeta potential decreased depending on time because of the aggregation of particles.

\section{CONCLUSION}

Ofloxacin, a second generation fluoroquinolone,

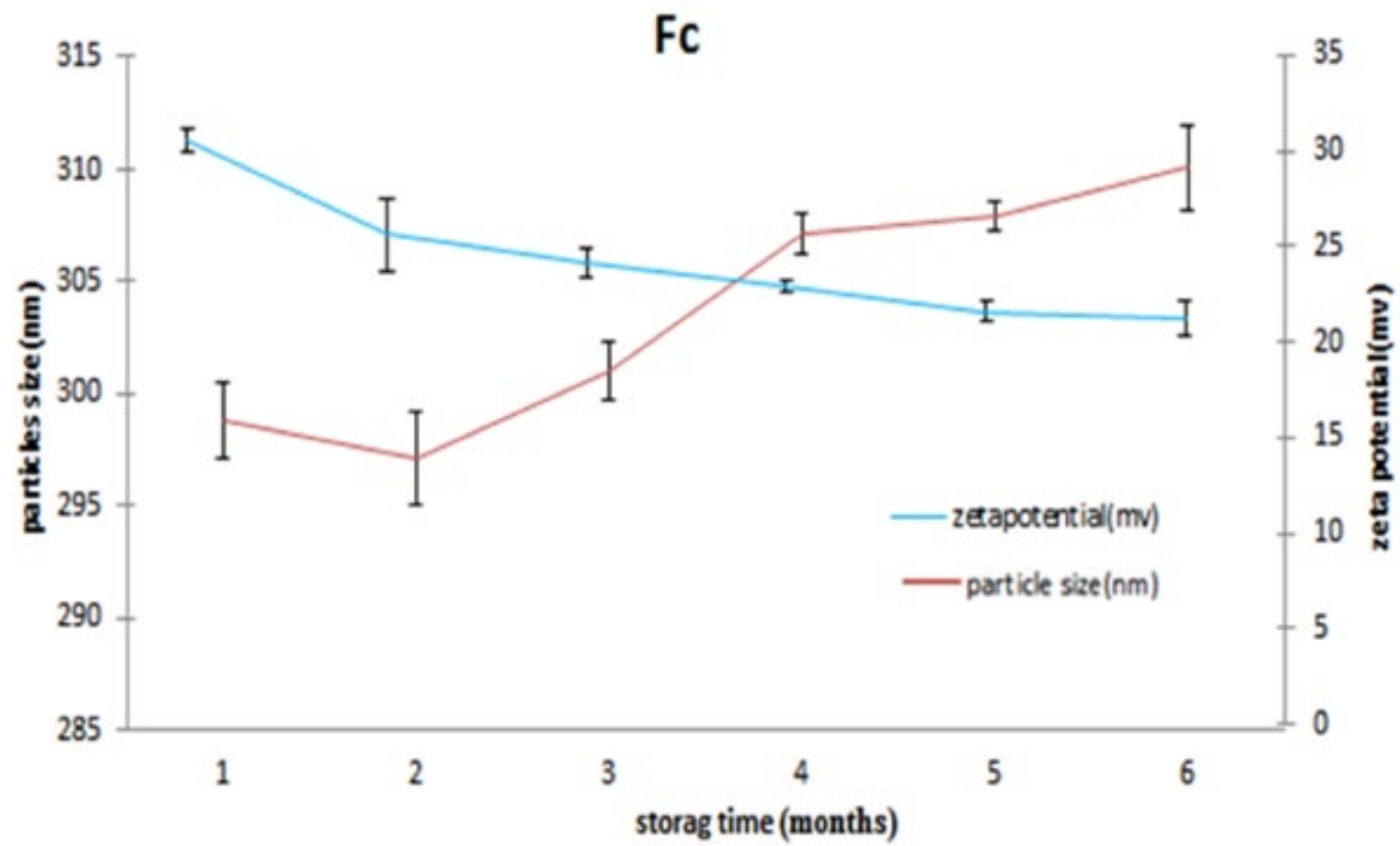

FIGURE 8 - Particle size and zetapotential of reconstituted Fc(chitosan/TPP nanoparticles) upon storage for 6 months at room temperature. 


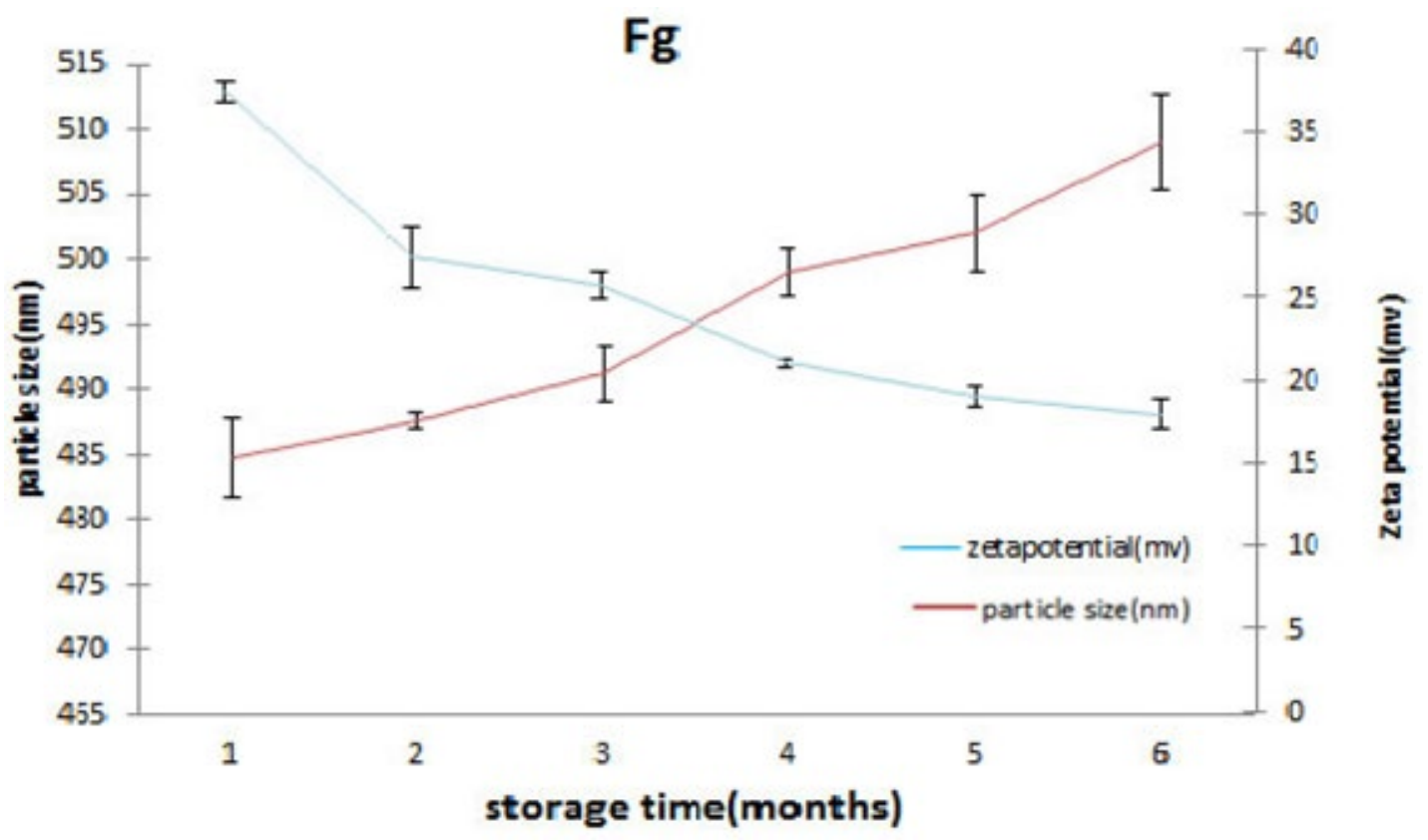

FIGURE 9 - Particle size and zetapotential of reconstituted Fg (chitosan/TPP-Alginate nanoparticles) upon storage for 6 months at room temperature.

and a broad-spectrum antibacterial agent were used in the treatment of ocular infections, and were successfully formulated in the form of CS/TPP and CS/TPP-ALG nanoreservoir systems.

Nanoparticles were prepared and characterized in the present study. Obtained nanoparticles had small particle size and positive surface charges, which improved good stability in six months. The NPs thus produced improved high penetration through isolated sheep cornea due to the interaction with negatively charged biological membranes and site-specific targeting in vivo. According to Calvo et al. (1994), the results suggest the ability of these colloidal carriers to specifically target drugs to the cornea while avoiding systemic drug loss through the conjunctiva. Also, nano-conjugated OFX showed an effective drug delivery system for penetrated biological cells (Calvo et al., 1994; Chakraborty et al., 2012). In conclusion, these coatings achieved pronounced penetration enhancing effect as compared to OFX solution (5-6.5 times). It is notable that the chitosan and chitosan/alginate coating, as biocompatible and biodegradable polymer, has the potential to be used as a non-toxic penetration enhancer in nanoparticle form, especially for posterior ocular drug delivery. In addition, the study has demonstrated a slower, sustained release of OFX through cornea, as compared to the solution formulation of this drug. Thus, this formulation of nanoparticles has a strong potential for a sustained release effect of the drug, when applied to the eye topically.

\section{ACKNOWLEDGEMENTS}

This work was financially supported by Research \&Technology Center of Kermanshah University of Medical sciences Iran, (Financial Cod 96363).

\section{REFERENCES}

Abraham S, Furtado S, Bharath S, Basavaraj BV, Deveswaran R, Madhavan V. Sustained ophthalmic delivery of ofloxacin from an ion-activated in situ gelling system. Pak J Pharm Sci. 2009;22(2):175-79.

Agnihotri SA, Aminabhavi TM. Chitosan nanoparticles for prolonged delivery of timolol maleate. Drug Dev Ind Pharm. 2007;33(11):1254-62.

Aspden TJ, Mason JD, Jones NS, Lowe J, Skaugrud O, Illum L. Chitosan as a nasal delivery system: the effect of chitosan solutions on in vitro and in vivo mucociliary transport rates in human turbinates and volunteers. J Pharm Sci. 1997;86(4):50913.

Bron AG, Leber G, Rizk SN, Baig H, Elkington AR, Kirkby GR, et al. Ofloxacin compared with chloramphenicol in the management of external ocular infection. Br J Ophthalmol. 1991;75(11):675-79. 
Calvo P, Alonso MJ, Vila-Jato JL, Robinson JR. Improved ocular bioavailability of indomethacin by novel ocular drug carriers. J Pharm Pharmacol. 1996;48(11):1147-52.

Calvo P, Remunan-Lopez C, Vila-Jato JL, Alonso MJ. Novel hydrophilic chitosan-polyethylene oxide nanoparticles as protein carriers. J Appl Polymer Sci. 1997;63(1):125-132.

Calvo P, Thomas C, Alonso MJ, Vila-Jato JL, Robinson JR. Study of the mechanism of interaction of poly ( $\epsilon$-caprolactone) nanocapsules with the cornea by confocal laser scanning microscopy. Int J Pharm. 1994;103(3):283-291.

Chakraborty SP, Sahu SK, Pramanik P, Roy S. In vitro antimicrobial activity of nanoconjugated vancomycin against drug resistant Staphylococcus aureus. Int J Pharm. 2012;436(12):659-676.

Das S, Suresh, PK. Drug delivery to eye: Special reference to nanoparticles. Int J Drug Deliv. 2010;2(1):12-21.

De Campos AM, Sánchez A, Alonso MJ. Chitosan nanoparticles: a new vehicle for the improvement of the delivery of drugs to the ocular surface. Application to cyclosporin A. Int J Pharm. 2001;224(1-2):159-168.

Di Colo G, Zambito Y, Burgalassi S, Nardini I, Saettone MF. Effect of chitosan and of N-carboxymethylchitosan on intraocular penetration of topically applied ofloxacin. Int $\mathbf{J}$ Pharm. 2004;273(1-2):37-44.

Duxfield L, Sultana R, Wang R, Englebretsen V, Deo S, Rupenthal ID, Al-Kassas R. Ocular delivery systems for topical application of anti-infective agents. Drug Dev Ind Pharm. 2016;42(1):1-11.

Fan W, Yan W, Xu Z, Ni H. Formation mechanism of monodisperse, low molecular weight chitosan nanoparticles by ionic gelation technique. Colloids Surf B Biointerfaces. 2012;90:21-27.

Felt O, Furrer P, Mayer JM, Plazonnet B, Buri P, Gurny R. Topical use of chitosan in ophthalmology: tolerance assessment and evaluation of precorneal retention. Int J Pharm. 1999;180(2):185-193.

Hirano S, Seino H, et al. Bio-compatibility of chitosan by oral and intravenous administrations. Abstracts of papers of the American Chemical Society. Washington, DC; 1988.
Lang JC. Ocular drug delivery conventional ocular formulations. Adv Drug Deliv Rev. 1995;16(1):39-43.

Maurice D, Mishima S. Ocular pharmacokinetics. Pharmacology of the Eye. Berlin Heidelberg: Springer; 1984. p.19-116.

Motwani SK, Chopra S, Talegaonkar S, Kohli K, Ahmad FJ, Khar RK. Chitosan-sodium alginate nanoparticles as submicroscopic reservoirs for ocular delivery: formulation, optimisation and in vitro characterisation. Eur J Pharm Bioph. 2008;68(3):513-525.

Nagarwal RC, Kant S, Singh PN, Maiti P, Pandit JK. Polymeric nanoparticulate system: a potential approach for ocular drug delivery. J Control Release. 2009;136(1):2-13.

Osato MS, Jensen HG, Trousdale MD, Bosso JA, Borrmann LR, Frank J, Akers P. The comparative in vitro activity of ofloxacin and selected ophthalmic antimicrobial agents against ocular bacterial isolates. Am J Ophthalmol. 1989;108(4):380-386.

Papageorgiou SK, Kouvelos EP, Favvas EP, Sapalidis AA, Romanos GE, Katsaros FK. Metal-carboxylate interactions in metal-alginate complexes studied with FTIR spectroscopy. Carbohydr Res. 2010;345(4):469-473.

Reimondez-Troitiño S, Csaba N, Alonso MJ, de la Fuente M. Nanotherapies for the treatment of ocular diseases. Eur J Pharm Biopharm. 2015;95(Pt B):279-293.

Sahoo S, Chakraborti CK, Behera PK. FTIR and Raman spectroscopic investigations of Ofloxacin/Carbopo1940 mucoadhesive suspension. Int J PharmTech Res. 2012;4(1):382391.

Sahoo SK, Dilnawaz F, Krishnakumar Sl. Nanotechnology in ocular drug delivery. Drug Discov Today. 2008;13(3):144-151.

Sechoy O, Tissié G, Sébastian C, Maurin F, Driot JY, Trinquand C. A new long acting ophthalmic formulation of carteolol containing alginic acid. Int J Pharm. 2000;207(1):109-116.

Singh J, Dutta P. Preparation, antibacterial and physicochemical behavior of chitosan/ofloxacin complexes. Int J Polymeric Mater. 2010;59(10):793-807.

Sultana Y, Jain R, Aqil M, Ali A. Review of ocular drug delivery. Curr Drug Deliv. 2006;3(2):207-217. 
Takeuchi H, Yamamoto H, Niwa T, Hino T, Kawashima Y. Enteral absorption of insulin in rats from mucoadhesive chitosan-coated liposomes. Pharm Res. 1996;13(6):896-901.

Thakur RR, Kashiv M. Modern delivery systems for ocular drug formulations: a comparative overview WRT conventional dosage form. Int J Res Pharm Biomed Sci. 2011;2:8-18.

Urtti A, Pipkin AD, ROrk JD, Sendo T, Finne U, Repta AJ. Controlled drug delivery devices for experimental ocular studies with timolol 2. Ocular and systemic absorption in rabbits. Int $\mathrm{J}$ Pharm. 1990;61(3):241-249.

Vårum KM, Myhr MM, Hjerde RJ, Smidsrød O. In vitro degradation rates of partially $\mathrm{N}$-acetylated chitosans in human serum. Carbohydr Res. 1997;299(1):99-101.
Wu Y, Yang W, Wang C, Hu J, Fu S. Chitosan nanoparticles as a novel delivery system for ammonium glycyrrhizinate. Int $\mathbf{J}$ Pharm. 2005;295(1):235-245.

Yan XL, Khor E, Lim LY. Chitosan-alginate films prepared with chitosans of different molecular weights. J Biomed Mater Res. 2001;58(4):358-365.

Received for publication on $13^{\text {th }}$ March 2017 Accepted for publication on $12^{\text {th }}$ June 2018 